\title{
IMPLEMENTATION OF A CRYPTO-STEGANOGRAPHIC SYSTEM BASED ON THE AES-CBC ALGORITHM
}

\section{EDWAR JACINTO GÓMEZ, HOLMAN MONTIEL ARIZA, FERNANDO MARTÍNEZ SANTA}

Facultad Tecnológica, Universidad Distrital Francisco José de Caldas, Colombia

ABSTRACT
Safeguarding information has been one of the oldest needs of mankind, as well as protecting privacy from malicious
attacks, thus steganography arises as a response to that need. Common steganography techniques, such as LSB, remove
the natural entropy of the least significant bits in an image, which can raise suspicions about the existence of hidden
information. The objective of this paper is to embed an AES-CBC encrypted secret message in an image using the LSB
method to eliminate any kind of patterns that may be generated when applying steganographic techniques, in addition
to providing double security to the information using a standard block cipher. A high-level software tool was developed
that works with image processing and the use of cryptographic libraries that have the standard of block cipher such as
AES-CBC. After this implementation, a series of tests were carried out with different sizes of images and information
to be ciphered, which measured the time it takes to perform the entire process of mixing and encryption of information,
thus it was possible to give a double security scheme to the information in terms of using LSB without generating
patterns easily detectable by any tool and making a basic and secure encryption scheme of information, all
simultaneously.
KEYWORDS: Steganography, Cryptography, LSB, AES-CBC algorithm.

Received: Jun 09, 2020; Accepted: Jun 29, 2020; Published: Sep 26, 2020; Paper Id.: IJMPERDJUN20201435

\section{INTRODUCTION}

The development of information and communication technologies(Lin et al., 2014) has created the need to exchange information secretly and hide the existence of the message (Mangela, 2017). To do this, cryptography(Agarwal \& Bisht, 2018) and steganography (Cao \& Jung, 2015; Shelke \& Jagtap, 2015) are used together, which adds a layer of security to the message in the event of a malicious attack (Morkel, 2015). Steganography and cryptography (Charan et al., 2015) aim to protect information but differ mainly in that the former technique hides data (Roy \& Changder, 2014) in such a way that it appears that there is no message (Alam, Zakariya, et al., 2014), while cryptography turns a plain text into one that is incomprehensible (Deshmukh \& Pattewar, 2015) to any unauthorized recipient even if a secret message is known to exist (Jakhar \& Saini, 2016).

In steganography (Guo et al., 2014; Rodyhin \& Fedorov, 2015) two basic files are required: the Stegoimage (Halder \& Mukherjee, 2016) or cover image which is where the information is entered (Su et al., 2018) and the message file to be hidden Stego-message (Prabakaran et al., 2014), although several methods have also been used to hide data in audio and video files (Hemalatha \& Maneesha, 2016; Vasoya et al., 2018). The LSB method (Kang \& Iwamura, 2014) has been one of the most used, which, consists of changing the least significant bit of each pixel of the Stego-image with a bit of information from the Stego-message (Alam, Kumar, et al., 2014; Kapgate \& Agrawal, 2018). 
But hiding messages within an image is not enough (Odeh et al., 2014) since if an attacker knows that there is a steganography message, he could infer with which methodology the message was hidden within the image and thus get to know the hidden information (Grover et al., 2018). For this it is important to complement steganography techniques with cryptography methods (Raniprima et al., 2017; Sharon et al., 2017) to prevent an external person from accessing the secret message (Seethalakshmi et al., 2016). As a result of this, it was decided to use AES as it is the most popular block symmetric encryption algorithm (Malathi et al., 2018), it consists of segmenting the data in blocks, and in addition to this, a key or password of the same size of the information block is needed. AES has different modes of operation, in terms of the use of the keys and their respective subkeys, among which is the CBC (Cipher Block Chaining) technique that segments the message in blocks of 128, 192 or 256 bits and uses an XOR operation to mix the umpteenth subkey of the previous block with the plain text of the current block; 9,11 or 13 iterations are needed depending on the degree of security of the algorithm.

Before starting the encryption process, the key exchange process must be carried out in such a way that information security is not compromised. To this end, it was decided to use a cryptography protocol that allows the exchange of keys anonymously, using an asymmetric encryption algorithm.

\section{METHODOLOGY}

A steganography application was proposed shown in Figure 1 that uses as a basic technique the LSB to hide a Stego-message or plain text to be sent, which, has been previously encrypted with AES-CBC, to avoid that the text is read in case the existence of the message is detected inside the image, all this making a safe exchange of the key and without altering any of the characteristics of the Stego-image.

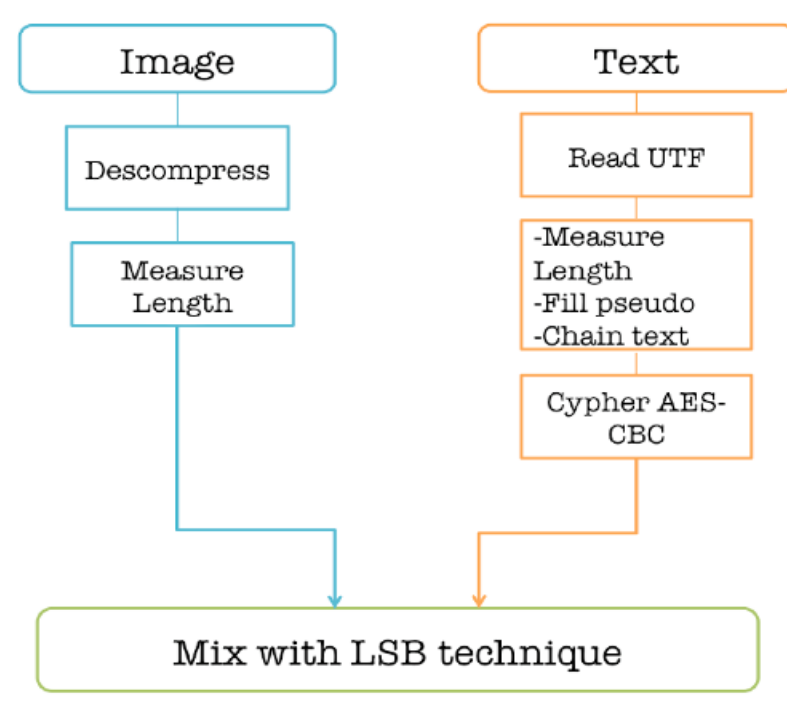

Figure 1: Description of the Proposed Method.

The combined scheme of mixing and encrypting the information should start with determining the best way to exchange the key by a secure means. Also, a way to delimit the information should be established by packaging the data with a specific character sequence, which indicates the exact position of the encrypted stemming message, as it is an encryption scheme that complies with the philosophy of Feistel networks by applying the reverse process, the message sent will be accessed hidden and encrypted, in a scheme that avoids the detection of hidden information, but if it is detected, it will maintain the integrity and security of the information. 
It is important to emphasize that the software tool will determine in a semi-automatic way the sizes of the Stegoimage concerning the size of the Stego-message be sent, in other words, if the message is short a lower resolution image will be required, but it will always be necessary to fill in the information with a sequence of pseudo-random characters, to avoid the LSB plane of the Stego-image being empty and in this way, the detection tools will show the presence of a message in the Stego-image.

\section{DEVELOPMENT AND IMPLEMENTATION}

In this section will describe the functions and parts of the software tool, which begins with the basic techniques of steganography and the necessary cryptography concepts to make the implementation

\subsection{LSB (LEAST SIGNIFICANT BIT) TECHNIQUE}

It is the most basic and therefore most widely used technique, in which plain text is converted into ASCII, this is then fed into a binary string and mixed into the least significant bit of text, as seen below, Figure 2:

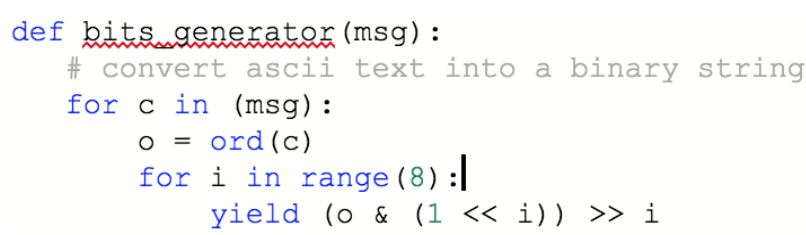

Figure 2: LSB algorithm, Chain Generation.

Initially, it is required to have installed the Opencv version 2 libraries, which allows operations with images and vectors, this library has some tools for digital signal and image processing. In this case, the function is defined: bits_generator, this function, is in charge of reading the message ( $\mathrm{msg}$ ) which was already read from a file that stores information in ASCIIUTF8 format, when reading the string of characters msg, the conversion of ASCII to its respective 8-bit value is performed with the command ord that gives the value in the whole format that corresponds to the value of the ASCII table of each of the data of the string of characters read, after this a string of binary numbers is created using a cycle that goes through each 8-bit data and puts it in a binary string with which it is ready to be mixed with each of the pixels of the chosen image to be the Stego-image, the RGB structure of the imaged is shown in Figure 3.

At this point, an algorithm must be made that somehow guarantees that the size of the Stego-message corresponds to the number of pixels that the image has, so to avoid limitations on the size of the message, the size of the image is preserved and the Stego-message is repeated as many times as necessary, the value of the rows and columns of the image is determined and the message to be mixed is repeated a whole number of times, if at the end the exact size of the message does not match, bits are included in a random pattern to complete each of the pixels in the image. To avoid confusion for the receiver of the message, some delimiters of the message are defined, that the message is repeated more than once in the image serves as a methodology to verify the information. 


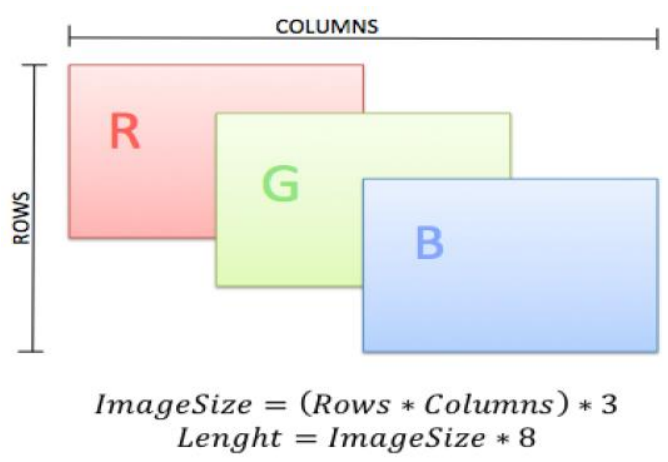

Figure 3: RGB Color Model.

\subsection{ENCRYPTION. (AES AND CBC)}

AES is an encryption standard that is a symmetrical cipher or a finite block cipher (128 bits). It consists of three main rounds: The initial round, the standard round and the final round. The initial round is based on the AddRoundKey transformation, which performs an XOR operation between two matrices, the state matrix (which is the one containing the blocks of the original text) and the key matrix.

After this, the standard round starts, this round consists of 4 processes: ByteSub, ShiftRow, MixColumns and AddRoundKey. ByteSub does the substitution of each of the bytes of the resulting array from the previous round, but this substitution depends on these same bytes. Using an inversion operation and a linear transformation that is carried out point by point to the matrix resulting from the initial round.

ShiftRows applies shifts to the rows of the state matrix, so the first row is shifted by 0 bytes, the second by 1 byte, and so on. MixColumns performs the function of taking each column and multiplying it by a constant matrix.

AES is applied in this way for encryptions up to 128 bits, but when there is a much longer message as in this case the input must be segmented into blocks. For this AES has among its modes of operation one called CBC (Cipher Block Chaining) that allows encrypting messages of greater length, its structure has shown in Figure 4.

CBC proposes to segment in blocks of fixed length the information and to the first segment to apply an XOR operation with a random number to the one that is denominated like initialization vector, this initialization vector can be zero although it is not recommended since it would reduce the security level of the algorithm. Having the result of this exercise is encrypted with a key and thus the first encrypted block is obtained. This way, the next block is applied XOR together with the previous ciphertext and is also coded for the following blocks.

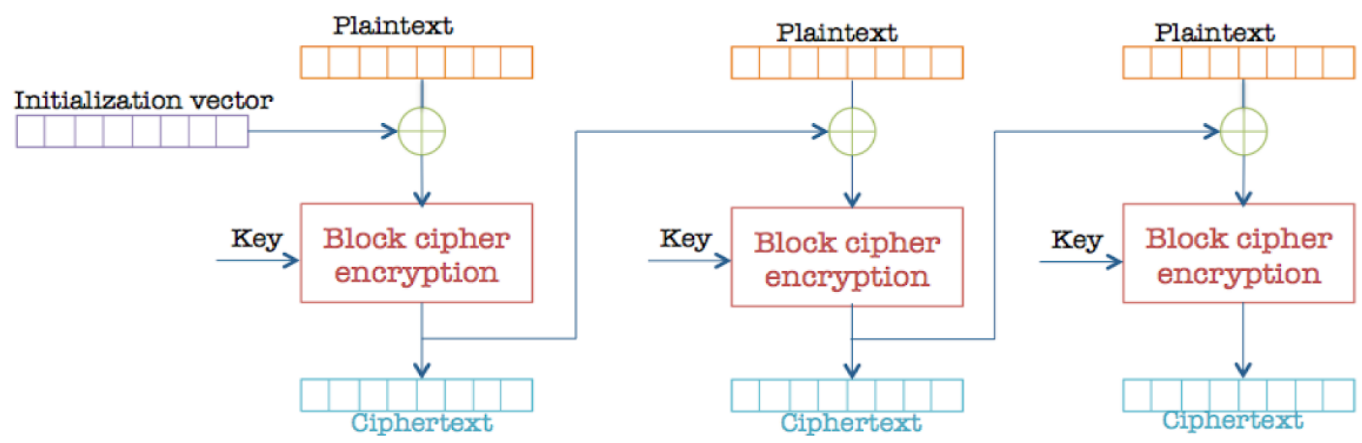

Figure 4: CBC Block diagram 


\subsection{APPLICATION DEVELOPMENT}

The process of mixing the information to be sent safely with the least significant bit of the image begins by decompressing the image from the source format to a basic matrix with its three respective RGB channels. At the same time, the string of characters of the message to be sent must be read secretly, ensuring that the characters are UTF-8 or compatible, if the message exceeds the number of pixels, then it will be required to choose an image with a higher resolution, the software tool designed will throw an error if this happens, after ensuring that the information can be contained sufficiently in the image, at this point it is important to note that in a stegano image of only 4 megapixels it is possible to "hide" all the Quixote of the Spot in the Least Significant Bit, so this check is purely to ensure that the indexing of the arrays is consistent and achievable, but on the other hand it must be guaranteed that the amount of bits of the characters of the information is exactly the one that gives the multiplication of the width and height of the image, in other words the amount of characters must be equal to the amount of pixels of the image, so a packing of the string is made and the length of the string is completed with a series of pseudo random characters within the alphabet space to guarantee that if a cryptographic analysis tool does not find spaces without information and in this way it can infer that there is hidden information in the Stego-image, after completing the string, the encryption is performed in AES-CBC, taking into account that this type of ciphers take as input a string of bits or a block of pure binary information, so the data conversions from string to pure binary string must be performed. Finally, each pixel is simply scrolled through, placing the least significant bit of the resulting binary string of the information to be encrypted in each pixel. Finally, the image is displayed and stored in a new file that will be transmitted securely.

The encryption process is necessary because the original image has a certain entropy of the least significant bit, this means that, if the least significant bit was taken of the image in which the message is to be introduced, the brightest areas tend to be 1 as can be seen in the following image, Figure 5:

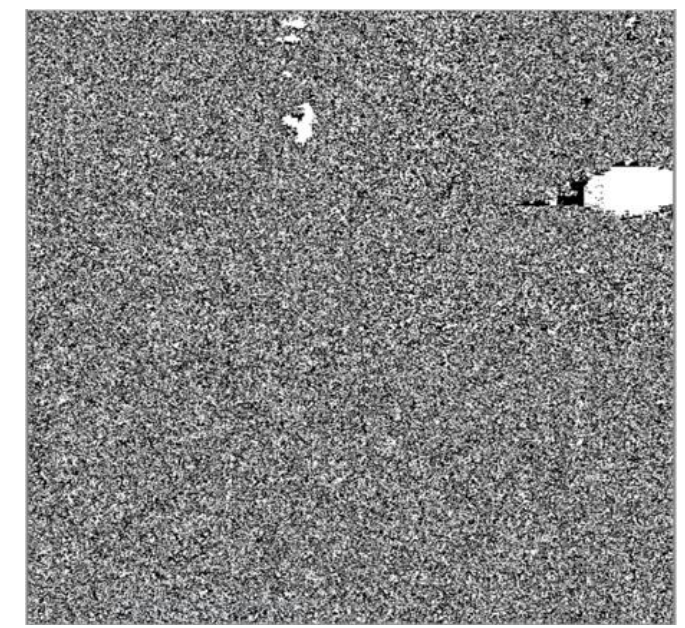

Figure 5: A Least Significant Bit of the Original Image (Entropy Analysis)

Whereas we can see there is a white area that matches the brightest part of the original image, after that, the process to mixed message with the image had been done, its less significant bits will no longer look the same as the originals, that bright part will be removed and that is where you can discover if the image has hidden information. In other words, the image loses its natural entropy and one may suspect a message hidden within the illustration by the notorious patterns that are generated, see Figure 6. 


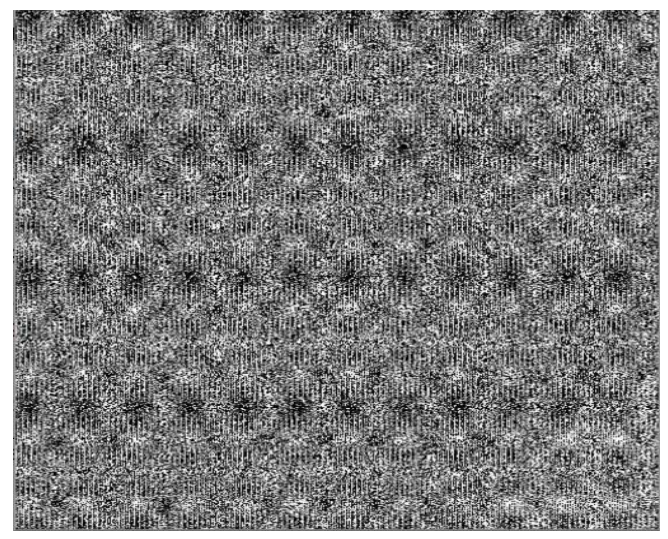

Figure 6: A Least Significant Bit of the image with Steganography and Visible Pattern

A complete explanation of the design process of the software tool is shown in a flow chart of the application, where each of the sub-processes necessary to perform the mixing of the encrypted information through AES-CBC with the LSB technique can see in the diagram corresponding to Figure 7.

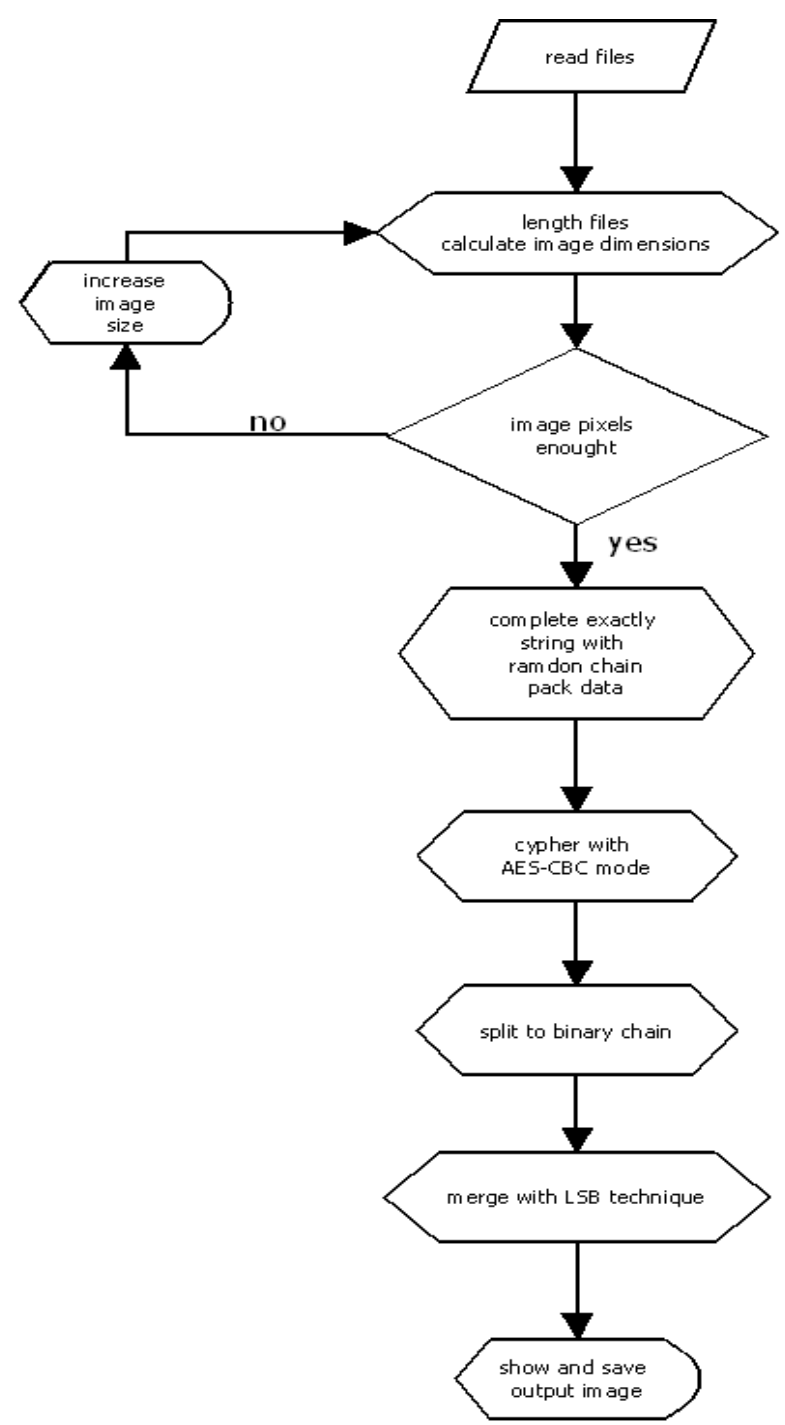

Figure 7: Algorithm Block Diagram 
Therefore, as an additional security layer encryption with AES and CBC is applied to eliminate those patterns that arise when embedding the Stego-message, as a result, is obtained an image with higher entropy and that has a high degree of security due to the encryption method used, as shown in Figure 8.

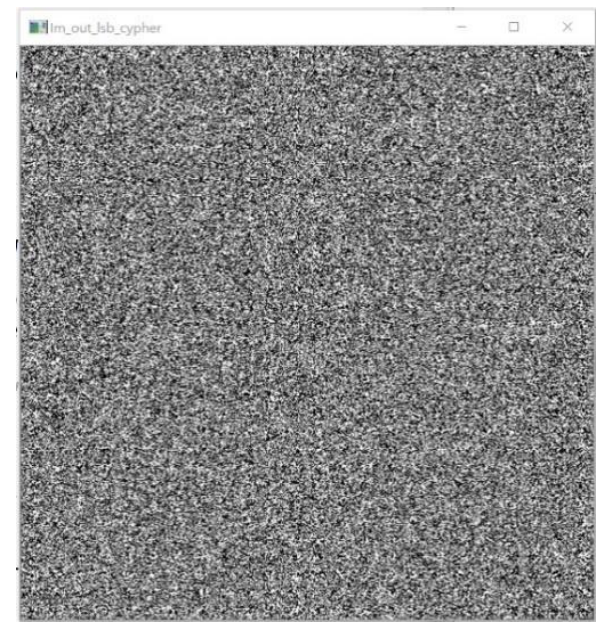

Figure 8: A least significant bit of the Encrypted Image

\section{RESULTS AND ANALYSIS}

Finally, it is important to make measuring of the performance of the algorithm, wherefore the tests with different lengths of the texts and images files were realized and the results were shown in Table 1.

Table 1: Time Measurement with the Proposed algorithm

\begin{tabular}{|c|c|c|c|}
\hline Archive & $\begin{array}{c}\text { Image size } \\
(\mathbf{K B})\end{array}$ & $\begin{array}{c}\text { Text size } \\
(\mathbf{K B})\end{array}$ & $\begin{array}{c}\text { Execution time } \\
\text { (Seg) }\end{array}$ \\
\hline 1 & 92 & 23 & 1,7 \\
\hline 2 & 115 & 47 & 3,48 \\
\hline 3 & 143 & 94 & 6,7 \\
\hline 4 & 413 & 188 & 9,9 \\
\hline 5 & 2400 & 376 & 37,46 \\
\hline 6 & 4314 & 1038 & 59,84 \\
\hline
\end{tabular}

\section{CONCLUSIONS}

A Python language method was implemented using standard image processing and cryptography libraries that hide a flat text file in UTF-8 format of any size and such information when hidden and combined with the LSB technique, does not generate significant changes in the output image, mixed with the text and also after passing it through the AES-CBC block cipher algorithm, which shows us that the input and output image after the cipher process have a high entropy so if the output image is encrypted with a tool to verify the existence of a hidden message it could go unnoticed.

The use of the LSB method is appropriate for the task of hiding information securely, but the secure sending of data is only guaranteed when using a standard cryptographic technique such as AES-CBC, which ensures that the secret is not violated if it is tried to break it. The sequential mixing of the key with the information by the CBC method guarantees a high complexity of the encryption algorithm and that it is not simply broken by any computational methods. 
By mixing two computer security techniques such as steganography and cryptography using a symmetrical block cipher, a double layer of security is given to the information by a method called mixed, named by some authors, which makes the sending of information safe and safeguards the secrecy of the data sent.

This is the first work of the research group SIE (Embedded Computer Security) and it opens the door for further work on the subject of steganography combined with classical cryptography since when measuring encryption times concerning the amount of information that needed to be hidden, it is noted that some of the processing times are excessive, so it is required to modify parts of the algorithm, verify if the algorithm and all the libraries can be run from the PC video card used and not from the CPU as it was currently run, another possible change or improvement would be to use cards with embedded Linux with dedicated use of video card for information processing or as an alternative solution, you can speed up the processing of the image using FPGA devices.

\section{REFERENCES}

1. Agarwal, S., \& Bisht, J. S. (2018). Improved BEMD data hiding scheme: Data hiding binary exploitation modification direction scheme using image steganography. International Conference on Recent Innovations in Signal Processing and Embedded Systems, RISE 2017, 2018-Janua, 206-211. https://doi.org/10.1109/RISE.2017.8378155

2. Alam, S., Kumar, V., Siddiqui, W. A., \& Ahmad, M. (2014). Key dependent image steganography using edge detection. International Conference on Advanced Computing and Communication Technologies, ACCT, 85-88. https://doi.org/10.1109/ACCT.2014.72

3. Alam, S., Zakariya, S. M., \& Akhtar, N. (2014). Analysis of modified triple - A steganography technique using Fisher Yates algorithm. 2014 14th International Conference on Hybrid Intelligent Systems, HIS 2014, $207-212$. https://doi.org/10.1109/HIS.2014.7086199

4. Cao, L., \& Jung, C. (2015). Combining Visual Saliency and Pattern Masking for Image Steganography. Proceedings - 2015 International Conference on Cyber-Enabled Distributed Computing and Knowledge Discovery, CyberC 2015, 320-323. https://doi.org/10.1109/CyberC.2015.95

5. Charan, G. S., Nithin Kumar, S. S. V., Karthikeyan, B., Vaithiyanathan, V., \& Divya Lakshmi, K. (2015). A novel LSB based image steganography with multi-level encryption. ICIIECS 2015 - 2015 IEEE International Conference on Innovations in Information, Embedded and Communication Systems, 1-5. https://doi.org/10.1109/ICIIECS.2015.7192867

6. Deshmukh, P. U., \& Pattewar, T. M. (2015). A novel approach for edge adaptive steganography on LSB insertion technique. 2014 International Conference on Information Communication and Embedded Systems, ICICES 2014, 978, 1-5. https://doi.org/10.1109/ICICES.2014.7033807

7. Grover, R., Yadav, D. K., Chauhan, D. K., \& Kamya, S. (2018). Adaptive steganography via image complexity analysis using $3 D$ color texture feature. 3rd International Conference on Innovative Applications of Computational Intelligence on Power, Energy and Controls with Their Impact on Humanity, CIPECH 2018, 125-129. https://doi.org/10.1109/CIPECH.2018.8724225

8. Guo, L., Ni, J., \& Shi, Y. Q. (2014). Uniform embedding for efficient JPEG steganography. IEEE Transactions on Information Forensics and Security, 9(5), 814-825. https://doi.org/10.1109/TIFS.2014.2312817

9. Halder, P., \& Mukherjee, I. (2016). A novel double layered image steganography in spatial domain using triangular number system. Proceedings of 2015 IEEE International Conference on Research in Computational Intelligence and Communication Networks, ICRCICN 2015, 411-416. https://doi.org/10.1109/ICRCICN.2015.7434274 
10. Hemalatha, R. K., \& Maneesha, P. (2016). Unified embedding method for color image steganography. 2016 International Conference on Information Communication and Embedded Systems, ICICES 2016, 1-5. https://doi.org/10.1109/ICICES.2016.7518872

11. Jakhar, D., \& Saini, G. (2016). Design of a novel regulated cascode current mirror. 2015 International Conference on Computing, Communication and Security, ICCCS 2015, 1-6. https://doi.org/10.1109/CCCS.2015.7374200

12. Kang, H., \& Iwamura, K. (2014). Image protection system with steganography and authentication. Proceedings - 201410 th International Conference on Intelligent Information Hiding and Multimedia Signal Processing, IIH-MSP 2014, 2(1), 467470. https://doi.org/10.1109/IIH-MSP.2014.123

13. Kapgate, A. R., \& Agrawal, S. S. (2018). Edge based data embedding steganography algorithm using ZedBoard. RTEICT 2017 - 2nd IEEE International Conference on Recent Trends in Electronics, Information and Communication Technology, Proceedings, 2018-Janua, 2203-2207. https://doi.org/10.1109/RTEICT.2017.8256991

14. Lin, C. Y., Wu, S. C., \& Wang, J. J. (2014). VQ image compression steganography based on section-based informed embedding. Proceedings - 2014 International Symposium on Computer, Consumer and Control, IS3C 2014, 111-114. https://doi.org/10.1109/IS3C.2014.40

15. Malathi, M., Rahul, M., Sathish Kumar, N., \& Thamaraiselvan, R. (2018). Enhanced image steganography using AES \& SPIHT compression. Proceedings of 2017 International Conference on Innovations in Information, Embedded and Communication Systems, ICIIECS 2017, 2018-Janua, 1-5. https://doi.org/10.1109/ICIIECS.2017.8276029

16. Mangela, S. (2017). Value Differencing.

17. Morkel, T. (2015). Self-sanitization of digital images using steganography. 2015 Information Security for South Africa Proceedings of the ISSA 2015 Conference, 1-6. https://doi.org/10.1109/ISSA.2015.7335073

18. Odeh, A., Elleithy, K., \& Faezipour, M. (2014). Fast real-time hardware engine for ZWC text steganography. 2014 4th International Conference on Wireless Communications, Vehicular Technology, Information Theory and Aerospace and Electronic Systems, VITAE 2014 - Co-Located with Global Wireless Summit, 1-5. https://doi.org/10.1109/VITAE.2014.6934454

19. Prabakaran, G., Bhavani, R., \& Sankaran, S. (2014). Dual wavelet transform used in color image Steganography method. Proceedings - 2014 International Conference on Intelligent Computing Applications, ICICA 2014, $193-197$. https://doi.org/10.1109/ICICA.2014.49

20. Raniprima, S., Hidayat, B., \& Andini, N. (2017). Digital image steganography with encryption based on rubik's cube principle. ICCEREC 2016 - International Conference on Control, Electronics, Renewable Energy, and Communications 2016, Conference Proceedings, 198-201. https://doi.org/10.1109/ICCEREC.2016.7814972

21. Rodyhin, M., \& Fedorov, O. (2015). Managing embedding parameters of DCT coefficient steganography algorithms subject to the given value of the JPEG quality factor. 2015 2nd International Scientific-Practical Conference Problems of Infocommunications Science and Technology, PIC S and T 2015 - Conference Proceedings, 1, $251-252$. https://doi.org/10.1109/INFOCOMMST.2015.7357327

22. Roy, R., \& Changder, S. (2014). Image steganography with block entropy based segmentation and variable rate embedding. 2014 2nd International Conference on Business and Information Management, ICBIM 2014, 75-80. https://doi.org/10.1109/ICBIM.2014.6970937

23. Seethalakshmi, K. S., Usha, B. A., \& Sangeetha, K. N. (2016). Security enhancement in image steganography using neural networks and visual cryptography. 2016 International Conference on Computation System and Information Technology for 
Sustainable Solutions, CSITSS 2016, 396-403. https://doi.org/10.1109/CSITSS.2016.7779393

24. Sharon, V., Karthikeyan, B., Chakravarthy, S., \& Vaithiyanathan, V. (2017). Stego Pi: An automated security module for text and image steganography using Raspberry Pi. Proceedings of 2016 International Conference on Advanced Communication Control and Computing Technologies, ICACCCT 2016, 978, 579-583. https://doi.org/10.1109/ICACCCT.2016.7831706

25. Shelke, S. G., \& Jagtap, S. K. (2015). Analysis of spatial domain image steganography techniques. Proceedings - 1st International Conference on Computing, Communication, Control and Automation, ICCUBEA 2015, 665-667. https://doi.org/10.1109/ICCUBEA.2015.136

26. Su, W., Ni, J., Li, X., \& Shi, Y. Q. (2018). A New Distortion Function Design for JPEG Steganography Using the Generalized Uniform Embedding Strategy. IEEE Transactions on Circuits and Systems for Video Technology, 28(12), 3545-3549. https://doi.org/10.1109/TCSVT.2018.2865537

27. Vasoya, D. L., Vekariya, V. M., \& Kotak, P. P. (2018). Novel approach for image steganography using classification algorithm. Proceedings of the 2nd International Conference on Inventive Systems and Control, ICISC 2018, Icisc, 10791082. https://doi.org/10.1109/ICISC.2018.8398970 